\title{
Covalent Scavengers for Primary and Secondary Amines
}

\author{
John C. Hodges* \\ Parke-Davis Pharmaceutical Research, 2800 Plymouth Road, Ann Arbor, MI 48105, USA \\ Fax (734) 622-3107; E-mail: Jack.Hodges@wl.com \\ Received 14 September 1999
}

new tools

\begin{abstract}
Covalent scavenging strategies which rely upon the chemical reactivity of primary and secondary amines have found application in the separation of amine starting materials from combinatorial synthesis products. The use of solid-supported scavengers, sequestration enabling reagents, and the technique of impurity annihilation all allow the removal of structurally diverse amines by procedures that are easily carried out in parallel and are amenable to automation.
\end{abstract}

$\begin{array}{ll}1 & \text { Introduction } \\ 2 & \text { Covalent Scavenging }{ }^{2} \\ 3 & \text { Covalent Scavengers for Amines } \\ 4 & \text { Conclusions and Outlook }\end{array}$

Key words: amines, automated synthesis, combinatorial chemistry, parallel purification, polymers

\section{$1 \quad$ Introduction}

Combinatorial organic synthesis is generally divided into two tactical domains, solid phase synthesis and solution phase synthesis. In the solid phase synthesis of a combinatorial library of isolated compounds, one has a choice of synthetic strategies. Parallel, spatially addressed formats may be used wherein each compound synthesized is located in a discrete reaction well. Alternatively, an encoded, mix and split strategy may be employed which minimizes the number of reaction vessels and reagent dispensing steps required. Either way, the solid support provides an inherently simple means for separation of the desired product from excess reactants by a simple filtration and rinsing of the solid-supported product with solvent. When the final product is cleaved from the support it is found in substantially pure form.

Solution phase approaches are limited to the parallel format. Furthermore, it is desirable to devise a common reaction work-up/purification strategy that can be applied with broad success to isolate each synthetic intermediate or final library product. With sufficient work-up/purification planning for each synthetic step, the final products can be isolated in a state of purity that is comparable to solid phase synthesis without time-consuming chromatography. Automation and parallel processing can reduce the tedium and human error associated with the repetitive manipulations of parallel synthesis and purification. The current state of the art in automation, however, is limited to the simple manipulations such as solution or slurry transfer and phase separations, including evaporation, filtration, liquid/liquid extraction, and solid/liquid extraction.
Primary and secondary amines are among the most popular classes of building blocks for combinatorial libraries of organic molecules. There are a wide variety of amines that are commercially available and nearly limitless numbers of novel amines that are otherwise accessible through short, robust syntheses. They are useful in a broad range of synthetic reactions and nitrogen-containing pharmacophores are important in many pharmaceutical and agricultural products. It is not surprising that carbon-nitrogen bond formation reactions are found in most of the synthetic routes to combinatorial libraries of organic molecules that have been described to date ${ }^{1}$. Convenient strategies for selective removal of primary and secondary amines will thus have broad applicability in the combinatorial synthesis field.

\section{Covalent Scavenging ${ }^{2}$}

The demand for simple and efficient separations that can be applied in parallel following solution phase syntheses have given rise to a number of practical purification strategies which can be easily automated. Scheme 1 generalizes several powerful parallel purification strategies that employ covalent scavengers to remove primary and secondary amines from desired products. In this generic reaction, a slight excess of the amine is used to consume all of reactant $\mathrm{X}$ and afford a maximal yield of the product, $R^{1} R^{2} N X$. To effect purification of this product, the residual amine is sequestered to the solid phase using one or

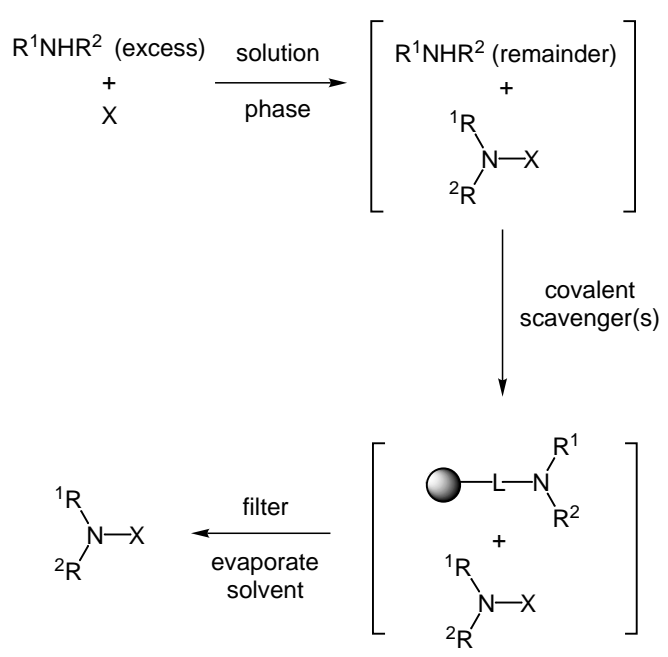

Scheme 1 
more insoluble and/or soluble scavenger reagents that are tailored to covalently react with primary or secondary amines. By virtue of the fact that the product lacks the chemical reactivity of a primary or secondary amine, it remains in solution where it can readily be isolated by a simple filtration to remove solid(s) and subsequent evaporation of the solvent(s).

The strength of covalent scavenging approaches for parallel purification lie in their inherent simplicity. Even though the set of amines used in a combinatorial array may have markedly different structures and physical properties, they all have a common chemical reactivity as nucleophiles. Hence a chemoselective strategy which employs electrophilic scavengers and uniformly sequesters all of the amine starting materials to the solid phase is easy to deduce. Furthermore, the choice of irreversible covalent reaction(s) removes equilibrium considerations from the separation process so that large excesses of the scavenging reagents are typically not required. Finally, it is a relatively straightforward matter to determine the modest excess of the scavenger reagent(s) required to readily overcome the frequent situation where variable residual amounts of individual starting amines occur from well to well.

The purification processes for amines described in Scheme 1 are chemically distinct from the time-honored and well-known concept of ion exchange in that covalent reactions rather than ionic interactions are used to sequester the amine. Ion exchange ${ }^{3}$ and covalent scavenging are both useful means for removal of amines in a parallel synthesis setting. The two methods are complementary. In a situation where it is desirable to remove any amine or quaternary ammonium salt, cation exchange resins may well be the sequestration reagent of choice. On the other hand, if one desires to selectively remove secondary and/or primary amines without removing tertiary amines or quaternary ammonium salts, covalent scavengers are preferred.
Another situation where covalent scavengers would be preferred over ion exchange resins exists when the desired product is sensitive to the strongly acidic groups typically found on cation exchange resins. Amine scavenging and sequestering strategies that combine covalent modification by soluble sequestration enabling reagents (SERs) with sequestration by anion exchange resins also have been reported ${ }^{2 \mathrm{c}, 4}$.

\section{Covalent Scavengers for Amines}

For practical reasons related to ease of automation and cost of reagents, the removal of amines by covalent scavenging involves a maximum of two synthetic transformations to the excess amine. Three types of strategies have been reported in the literature to date:

\section{- Solid-supported scavengers \\ - Sequestration enabling reagent (SER) plus solid- supported scavenger \\ - Impurity annihilation}

Solid-supported scavengers provide the simplest means of sequestering primary and secondary amines since they require only a single synthetic transformation. Several resin reagents have been described in the literature to date. Electrophilic scavenging functionalities used on these resins include isocyanate, isothiocyanate, carboxylic acid chloride, isatoic anhydride and aldehyde. Illustrative examples of the utility of amine scavenger resins in epoxide opening, alkylation, acylation, and reductive amination reactions are provided in the Table.

4-Isocyanatophenyl-methyl-poly(styrene-divinylbenzene) resin, 1, was first reported in the late 1970 s as a solid-supported amine scavenger in the patent literature by Patchornik, who made passing reference to its ability to quantitatively remove benzylglycine from chloroform

\section{Biographical Sketch}

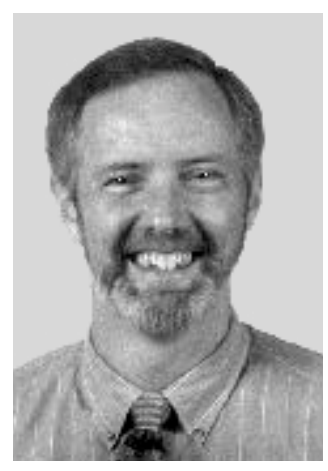

John C. Hodges was born in 1955 in Cumberland, MD. As a teen he moved to Wilmington, DE where he completed high school. $\mathrm{He}$ attended The University of Delaware where he received his Bachelors degree in Chemistry in 1977. Subsequently he attended The University of Arizona where he studied the synthesis and antineoplastic activity of mitosene analogs of the Mitomycin antitumor antibi- otics under the guidance of Prof. William A. Remers, receiving his Ph.D. in Pharmaceutical Chemistry in 1981 . His interest in antitumor agents continued during a postdoctoral fellowship with Prof. Andrew S. Kende at The University of Rochester where he worked on synthetic approaches to Taxusin. In 1982 he joined the research staff at Parke-Davis Pharmaceutical Research in Ann Arbor, MI where he has continued for seventeen years, working in a variety of therapeutic areas. For the past three and a half years he has held the position of $\mathrm{Sr}$. Director, Combinatorial Chemistry. Outside of work he can be found riding his motorcycle in fair weather and skiing when there is snow. Other personal interests include carpentry and cooking. 
solution ${ }^{5}$. The usefulness of this resin as a tool for purification remained largely unappreciated until nearly twenty years later when research groups at Eli Lilly ${ }^{6}$ and ParkeDavis ${ }^{7}$ reaffirmed the applicability of isocyanate resins, in this case, isocyanatomethyl polystyrene resin $\mathbf{2}$, as a means of removing primary and secondary amines from crude combinatorial synthesis products. The latter isocyanate resin is prepared by reaction of aminomethylpoly(styrene-divinylbenzene) with phosgene ${ }^{8}$ or triphosgene $^{7}$ (Scheme 2). The use of $\mathbf{2}$ for scavenging of amines in combinatorial synthesis applications has received considerable attention in the recent literature ${ }^{2,6,7}$. The analogous isothio-cyanatomethyl resin has not yet been reported as an amine scavenger but should have similar application?

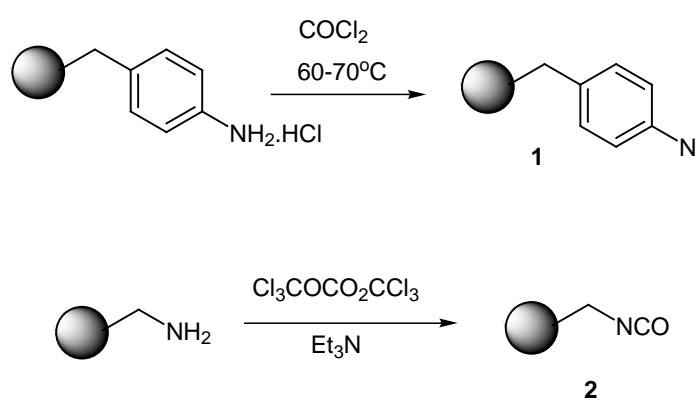

Scheme 2

Two other resins, $\mathbf{3}$ and $\mathbf{4}$, which bear a high loading of isocyanate groups and are likely to be useful as amine scavengers are shown in Schemes 3 and 4. These are both prepared by suspension polymerization of isocyanatecontaining monomers. Resin $\mathbf{3}$ is prepared by co-polymerization of methacryloyloxyethyl-isocyanate and divinylbenzene as a suspension in a perfluorocarbon solvent ${ }^{10}$. The perfluorocarbon solvent FC77 is preferred over water, which is the traditional solvent for suspension polymerization, since the isocyanate monomer is unstable to water at the polymerization temperature. Alternatively, 3-isopropenyl- $\alpha, \alpha$-dimethylbenzyl-isocyanate $\quad\left(\mathrm{TMI}^{\circledR}\right)$ may be co-polymerized with styrene in the presence of TEMPO resin as a solid-supported initiator to afford resin 4 which has a high isocyanate loading ${ }^{11}$. The result is that a large, isocyanate-functionalized bead is grown from a small initiator bead via a living free radical polymerization. Depending on one's perspective, this form of suspension polymerization either requires no suspending solvent or utilizes the monomers as both reactants and suspending solvents. Either way the potential water sensitivity of TMI ${ }^{\circledR}$ during polymerization is avoided since water is not used as the suspending solvent.

Carboxylic acid chloride on polystyrene resin (5) is a second type of solid-supported amine scavenger ${ }^{6 a}$. Compared to the isocyanate resins above, it has the inherent disadvantage that $\mathrm{HCl}$ is generated during the scavenging pro-

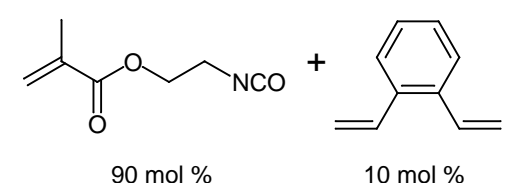

FC77

AIBN, dispersant Stir @ 250-300 rpm $50-55^{\circ} \mathrm{C}, 8 \mathrm{~h}$

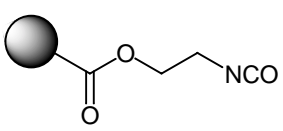

3

$5.2 \mathrm{mmol} \mathrm{N} / \mathrm{g}$

Scheme 3

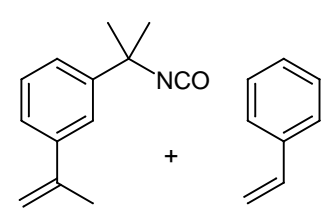

$\begin{array}{cc}\text { TMl } \\ 65 \mathrm{~mol} \% & 35 \mathrm{ST} \\ \mathrm{mol} \%\end{array}$

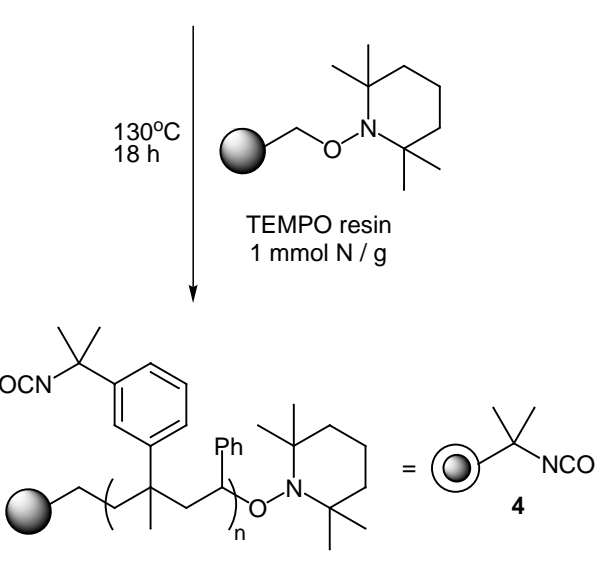

$2.5 \mathrm{mmol} \mathrm{NCO} / \mathrm{g}$

Scheme 4

cess. Thus a strategy for $\mathrm{HCl}$ removal is also necessary. There may be instances where the reactivity of an acid chloride would be preferred over an isocyanate. The acid chloride resin $\mathbf{5}$ is prepared by treatment of benzoic acid resin with thionyl chloride ${ }^{12}$ or oxalyl chloride ${ }^{13}$ (Scheme $5)$. 


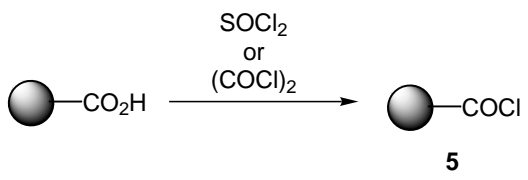

Scheme 5

A third class of polystyrene resin that has been reported as a scavenger of primary and secondary amines is the isatoic anhydride resin, 6. This resin is simply prepared by reacting the sodium salt of isatoic anhydride with a high loading Merrifield resin ${ }^{14}$ (Scheme 6). Isocyanate resin and isatoic anhydride resins appear to have roughly equal reactivity with primary and secondary amines and are essentially interchangeable. The ease of preparation of a high loading isatoic anhydride resin from inexpensive starting materials results in a significant price advantage per mmol relative to the lower loading isocyanatomethyl resin 9 .

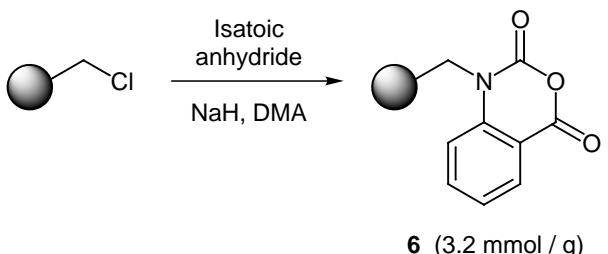

Scheme 6

Aldehyde polystyrene resins are a final chemical class of solid-supported amine scavengers that offer an alternative to acylation in the scavenging reaction. They can be used to selectively scavenge primary amines via imine formation. This makes them useful for separation of primary amine starting materials from secondary or tertiary amine products following a reductive amination (Table). Compared to the amine acylating resins described above, aldehyde resins are subject to more facile reversal of the covalent scavenging reaction since water can readily hydrolyse the imine and thereby liberate offending amines from the scavenger resin. Practically speaking this is not a major difficulty, especially if an excess of the aldehyde resin is used. The synthetic routes to aldehyde resins are shown in Scheme 7. Merrifield resin is readily oxidized to benzaldehyde resin 7 by heating with DMSO and sodium bicarbonate ${ }^{15}$. Alternatively aldehyde resin 8, also known as "Wang aldehyde resin", may be prepared either by oxidation of Wang resin ${ }^{16}$ or by reacting 4-hydroxybenzaldehyde with Merrifield's resin in the presence of a base ${ }^{17}$. An isomeric relative of Wang aldehyde resin (9), which is similarly prepared by reacting 3-hydroxybenzaldehyde with Merrifield's resin, can also be used ${ }^{18}$. Two examples of covalent scavenging with aldehyde resins following reductive amination ${ }^{6 a, 19}$ are shown in the Table.

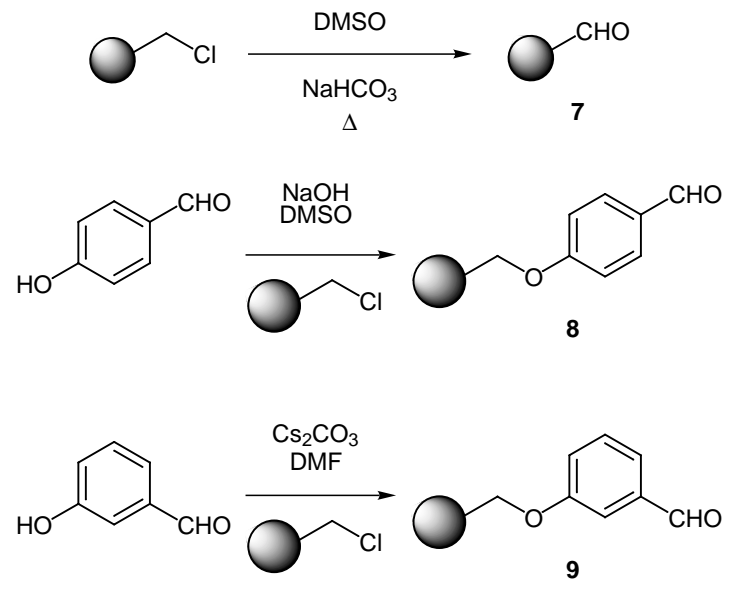

Scheme 7

As an alternative to electrophilic scavenger resins, removal of primary and secondary amines may also be effected in two reaction steps:1) Derivatization of the amine with an excess of a soluble bis-electrophile and 2) sequestration of the resulting amine derivative and remaining biselectrophile with a larger excess of a nucleophilic scavenging resin. This strategy has been effectively employed with hexafluoroisopropyl oxalate (10) as the SER ${ }^{20}$ (Scheme 8). In this process 10 first reacts with the amine starting material to form a hemi-amide which is subsequently sequestered by reaction with the supported amine, 11, which also covalently binds any remaining $\mathbf{1 0}$. The hexafluoro-isopropanol that is liberated in the process is volatile and easily evaporated with solvents. In addition to the added complexity of two reagent addition steps for the two scavenger reagents and two quantitative covalent reactions, this procedure has the inherent danger of producing a symmetrical oxalamide which would be an impurity that is likely to be difficult to separate from the desired product. However, with careful attention to the stoichiom-

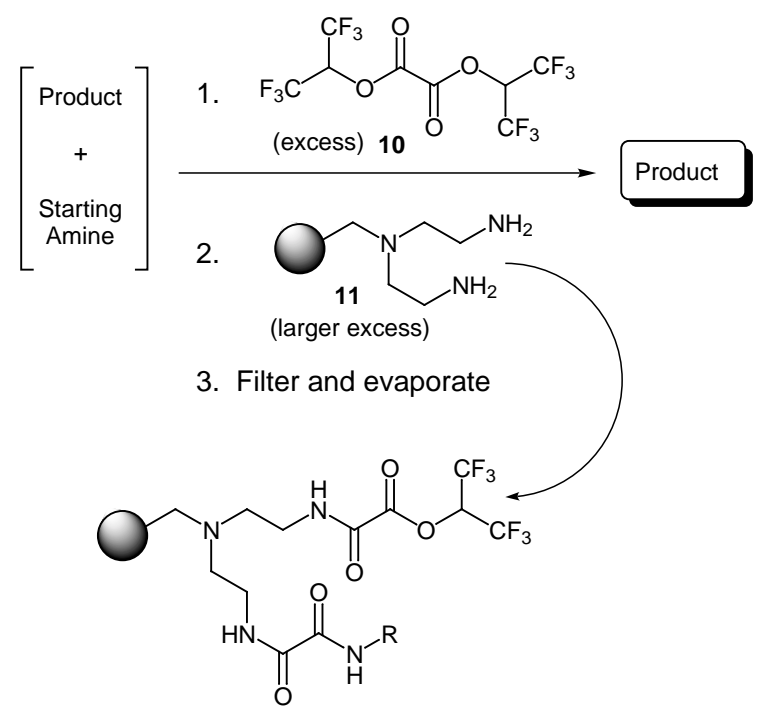

Scheme 8 
etry and order of addition, it is a useful alternative to onestep scavenging with an electrophilic resin. A number of commercially available bis-electrophiles, including bisisocyanates and bis-aldehydes, could potentially serve as alternative SERs that, when paired with an amino resin, would also be effective covalent scavenger systems for primary and secondary amines. Table 1 shows a selected example of this two-step solution and solid phase scavenging technique.

The latest addition to the armamentarium of methods for covalent scavenging of amines is a technique called "impurity annihilation" 21 (Scheme 9). Two scavenging reagents are also required for impurity annihilation but in this case they are both soluble. Addition of a solution of benzene-1,4-di-isocyanate (12) followed a brief time later by a solution of tetraethylenehexamine (13) to a crude reaction product that contains a primary or secondary amine starting material results in the preparation of a crosslinked urea polymer. The amine starting material is consumed by the di-isocyanate and the resulting benzene-1urea-4-isocyanate is covalently entrained during the polymerization reaction, making chain-terminating ureas. The highly cross-linked polyurea that is formed precipitates quantitatively from solution. Upon filtration and evaporation, product that is free of both the amine and the two monomers is isolated. This method is quite analogous to the stepwise addition of a SER and an amino polymer. However with impurity annihilation, one adds a polyamine instead of an amino-polymer and the insoluble support is created during the scavenging process, not before. The first step of the annihilation method also contains the inherent danger of creating soluble impurities by reaction of the di-isocyanate with two equivalents the amine to be scavenged. However if the prescribed stoichiometry and excesses of reagents are used, the production of symmetrical benzene-1,4-di-ureas can be avoided. One particular advantage of impurity annihilation is that both monomers are added as solutions and hence the process should be very easy to automate with liquid handling robots. Another advantage is cost. Since both scavenging monomers are common components of polymeric adhesives, they are very inexpensive compared to polymersupported scavenger reagents.

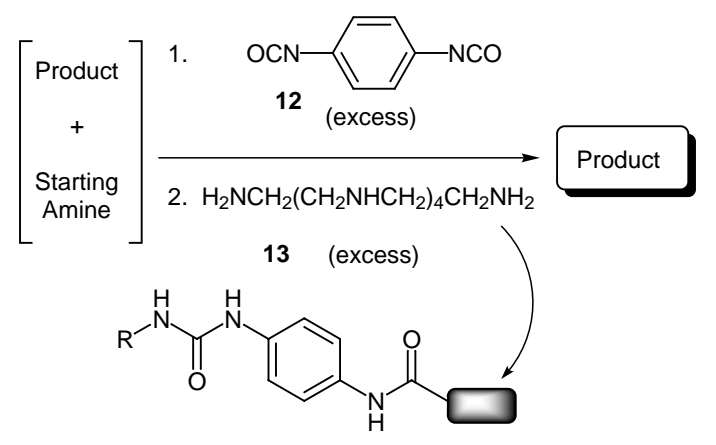

Scheme 9

\section{4}

\section{Conclusions and Outlook}

A variety of covalent scavenging methods for the selective separation of secondary and/or primary amine starting materials from desired synthetic products are now available. The inherent simplicity and generality of the methods makes them suitable for parallel application and amenable to automation. Thus they are of particular value to solution phase combinatorial synthesis since they avoid tedious and time-consuming chromatography. Additionally they may be useful in conventional organic synthesis in situations where an amine starting material has similar physical properties to the desired product and is difficult to remove by traditional means such as extraction, distillation, crystallization and chromatography.

Covalent scavenger resins are perhaps the most commonly employed tools today since they require only a single covalent reaction to effect a separation. Numerous polystyrene-based electrophilic scavenger resins with a range of reactivity toward secondary and/or primary amines have recently become commercially available ${ }^{22}$. Future extensions of these tools are likely to involve solid supports other than polystyrene. For example, covalent scavengers supported on silica, glass, and polypropylene may provide solvent compatibility or reactivity advantages over polystyrene resins in certain applications. Additionally, solid-supported scavengers in a variety of other forms such as wells, rods, pellets, tubing, etc. may eventually displace some of the resin bead scavengers in use today since dispensing and removing them with automation would be simpler than dispensing and filtering resin slurries. Two-step covalent scavenging protocols which either employ SERs with a solid supported scavenger or impurity annihilation monomers will likely gain greater acceptance as more examples appear in the literature since these reagents frequently have cost advantages over electrophilic scavenger resins and yet the methods are still easily automated.

\section{References and Notes}

(1) For recent reviews on combinatorial synthesis of organic molecules see: (a) Dolle, R.E.; Nelson, Jr., K.H. J. Combinatorial Chem. 1999, 1, 235. (b) Parlow, J.J.; Devraj, R.V.; South, M.S. Curr. Opin. Chem. Biol. 1999, 3, 320. (c) Booth, S.; Hermkens, P.H.H.; Ottenhdijm, H.C.J.; Rees, D.C. Tetrahedron 1998, 54, 15385. (d) Annual Reports in Combinatorial Chemistry and Molecular Diversity, Vol. 1 (1997), Moos, W.H.; Pavia, M.R.; Kay, B.K.; Ellington, R.B., Eds.; Vol 2 (1999), Moos, W.H.; Pavia, M.R., Eds.; Kluwer Academic Publishers: Dordrecht.

(2) For recent reviews on scavenger methods in parallel purification see: (a) Curran, D.P. Angew. Chem. Int. Ed. 1998, 37, 1174. (b) Gayo, L.M. Biotechnol. Bioeng. 1998, 61, 95. (c) Flynn, D.L.; Devraj, R.V.; Naing, W; Parlow, J.J.; Weidner, J.J.; Yang, S. Med. Chem. Res. 1998, 8, 219. (d) Booth, R.J.; Hodges, J.C. Acc. Chem. Res. 1999, 32, 18.

(3) (a) Siegel, M.G.; Hahn, P.J.; Dressman, B.A.; Fritz, J.E.; Grunwell, J.R.; Kaldor, S.W. Tetrahedron Lett. 1997, 38, 3357. (b) Gayo, L.M.; Suto, M.J. Tetrahedron Lett. 1997, 38 , 513. 
Table

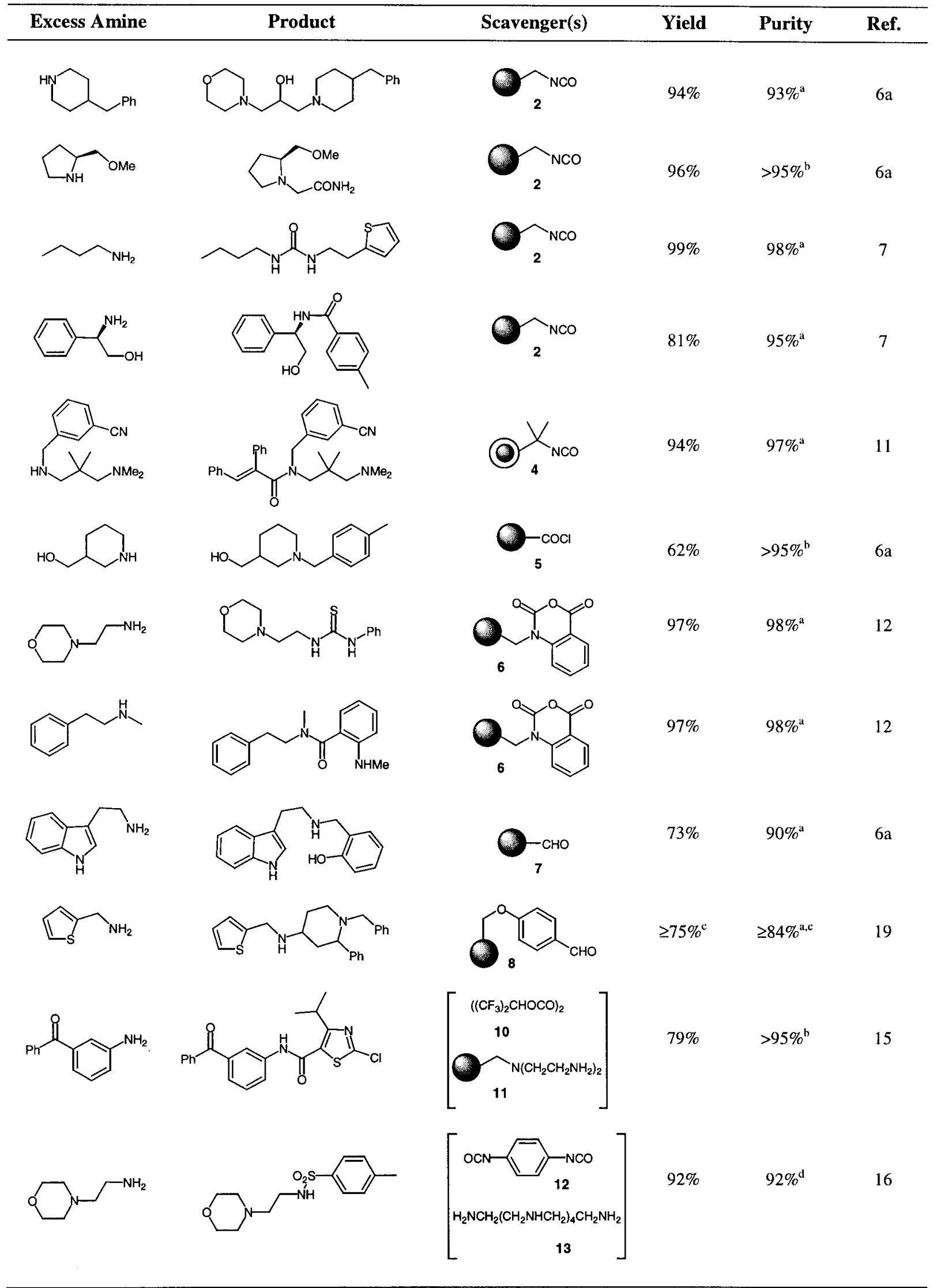

(a) purity estimated by HPLC (b) purity estimated by NMR (c) \% purity and \% yield after subsequent acylation by thiophene-2carboxylic acid chloride (d) purity estimate by GCMS and NMR. 
(4) (a) Parlow, J.J.; Flynn, D.L. Tetrahedron 1998, 54, 4013. (b) Parlow, J.J.; Niang, W.; South, M.S.; Flynn, D.L. Tetrahedron Lett. 1997, 38, 7959. (c) Flynn, D.L.; Crich, J.Z.; Devraj, R.V.; Hockerman, S.L.; Parlow, J.J.; South, M.S.; Woodard, S. J. Am. Chem. Soc. 1997, 119, 4874.

(5) Patchornik, A.; Warshawsky, A.; Fridkin, M.; Kalir, R. United States Patent 4,085,261; April 18, 1978 (Example 3).

(6) (a) Kaldor, S.W.; Siegel, M.G.; Fritz, J.E.; Dressman, B.A.; Hahn, P.J. Tetrahedron Lett. 1996, 37, 7193. (b) Dressman, B.A.; Singh, U.; Kaldor, S.W. Tetrahedron Lett. 1998, 39, 3631.

(7) Hodges, J.C.; Booth, R.J. J. Am. Chem. Soc. 1997, 119, 4882.

(8) Rebek, J.; Brown, D.; Zimmerman, S. J. Am. Chem. Soc. 1975, 97, 4407.

(9) The Combinatorial Chemistry Catalog, March 1999, pp. 1-3, S63, S66-7, Novabiochem Corp.

(10) Zhu, D-W. Macromolecules 1996, 29, 2813.

(11) Hodges, J. C.; Harikrishnan, L. S.; Ault-Justus, S. J. Combinatorial Chem., 2000, 2(1) in press.

(12) Leznoff, C.C.; Dixit, D.M. Can. J. Chem. 1977, 55, 3351.

(13) (a) Agrawal, Y.K.; Kaur, H.; Menon, S.K. React. Funct. Polym. 1999, 39, 155. (b) Meyers, H.V.; Dilley, G.J.; Durgin, T.L.; Powers, T.S.; Winssinger, N.A.; Shu, H.; Pavia, M.R. Molecular Diversity 1995, $1,13$.
(14) Coppola, G.M. Tetrahedron Lett. 1998, 39, 8233.

(15) (a) Beebe, X.; Schore, N.E.; Kurth, M.J. J. Am. Chem. Soc. 1992, 114, 10061. (b) Frechet, J.M.; Schuerch, C. J. Am. Chem. Soc. 1971, 93, 492. (c) Ayres, J.T.; Mann, C.K. J. Polym. Sci., Polym. Lett. Ed. 1965, 3, 505.

(16) Reggelin, M.; Brenig, V. Tetrahedron Lett. 1996, 37, 6851.

(17) Beebe, X.; Chiappari, C.L.; Olmstead, M.M.; Kurth, M.J.; Shore, N.E. J. Org. Chem. 1995, 60, 4204.

(18) Bolton, G.L.; Booth, R.J.; Creswell, M.W.; Hodges, J.C.; Warmus, J.W.; Wilson, M.W.; Kennedy, R.M. PCT International Patent Application WO 97422301997.

(19) Creswell, M.W.; Bolton, G.L.; Hodges, J.C.; Meppen, M. Tetrahedron 1998, 39, 1121.

(20) Parlow, J.J.; Mischke, D.A.; Woodard, S.S. J. Org. Chem. 1997, 62, 5908.

(21) Barrett, A.G.M; Smith, M.L.; Zecri, F.J. Chem. Commun. 1998, 21, 2317.

(22) Examples of commercial suppliers of covalent scavenger resins include Novabiochem Corp., Argonaut Technologies, Inc. and Aldrich Chemical Co.

Article Identifier:

1437-2096,E;2000,0,01,0152,0158,ftx,en;T01199ST.pdf 